BNL -43419

DE90 003700

Submitted to SRIB8

CON1 $-80081 \%$

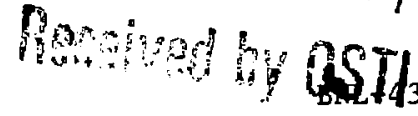

\title{
Soft X-Ray Imaging with the 35 Period Undulator at the NSLS
}

C. Buckley, H. Rarback, R. Alforque, D. Shu

NSLS, Brookhaven National Laboratory, Upton NY 11973 USA

H. Ade, S. Hellman, N. Iskander, J. Kirz, S. Lindaas, I. Mcnulty, M. Oversluizen, E. Tang Physics Department, SUNY at Stony Brook, Stony Brook NY 11794 USA

D. Attwood, R. DiGennaro, M. Howells, C. Jacobsen, Y. Vladimirsky

Center for X-ray Optics, Lawrence Berkeley Laboratory, Berkeley CA 94720 USA

S. Rothman

Center for X-ray Optics, Lawrence Berkeley Laboratory, and University of California, San Francisco, CA 94143.

D. Kern, D. Sayre

IBM Research Center, Yorktown Heights, NY 10598 USA

\section{Introduction}

Prior to the Phase II shutdown at the National Synchrotron Light Source (NSLS), in March, 1987, a 10-period mini-undulator was used for soft $x$-ray imaging on the temporary $\mathrm{X}-17 \mathrm{~T}$ beamline for six months [1]. During this brief period we gained considerable experience in the use of undulator radiation for high brightness applications, including the use of high power optics [2], and position feedback [3]. We commissioned a new scanning microscope [4], using high resolution zone plates [5], and a fast laser interferometer [6]. The microscope made it possible to study zymogen granules (subcellular structures which play an important role in the secretion of digestive enzymes in the pancreas) in an unaltered state: whole, unfixed, unstained and suspended in water. The first images displayed a distribution of material different than that seen in electron microscopy [7]. In addition, fixed and air dried zymogen granules were the subject of Gabor holography [8].

During the Phase II shutdown we made major changes to the entire system. At the present time the installation and commissioning of the new permanent line at beamline $\mathrm{X}-1 \mathrm{~A}$ is at an advanced stage. The design, performance and the projected experimental program of this facility is the subject of this paper.

\section{The Experimental Program}

The high brightness of the Soft X-Ray Undulator (SXU) operating on the X-Ray Ring makes it an ideal source for experiments that require high coherent flux. The imaging program is an excellent match to these capabilities. Coherent illumination of zone plates is required to achieve diffraction limited focusing of the beam. The microprobe formed this way will be used in two separate programs: scanning transnission microscopy and scanning photoemission microscopy. Coherence is also a critical ingredient in those imaging schemes that involve the detection of the diffracted radiation, such as Gabor holography, Fourier Transform holography, and soft $x$-ray diffraction The wavelength range of interest is 10 


\section{Submitted to SRI88}

to 50 Angstroms; the spectral resolving power $(\lambda / \delta \lambda)$ should be variable between 200 and 1800 .

Two experimental stations will be set up for imaging. One station will use $\mathrm{x}$ rays predominantly in the wavelength range 16 to 25 Angstroms; the other will use the range 20 to 50 Angstroms. Two zone plate based scanning microscopes are being installed, one on each branch of the beamline. The scanning transmission microscope uses the long wavelength branch. The beam traverses a 1200 Angstrom thick silicon nitride vacuum window before striking the zone plate, which focuses the beam onto the specimen. The zone plate and the specimen are in an atmospheric environment. The specimen is scanned under computer control, using piezoelectric flexure elements, while the transmitted $x$ rays are detected by a flow proportional counter. This microscope will primarily be used for biological investigations, although it is also suitable for the study of a wide variety of microstructures [9].

The scanning photoemission microscope will use the short wavelength branch. The beam will pass down the axis of a Cylindrical Mirror Analyzer (CMA) before reaching the zone plate, which focuses the beam onto the surface under study. The entire apparatus will be in a UHV environment. Electrons emitted from the surface will be energy analyzed by the CMA, and the specimen will be scanned using a stepper motor driven flexurehinged stage. This instrument is designed to provide moderate resolution $(\Delta \mathrm{E} \approx \mathrm{leV})$ $x$-ray photoemission spectroscopy at a spatial resolution of 0.15 microns for the study of heterogeneous surfaces primarily of interest to the material sciences [10].

The beamline section just upstream of the photoemission microscope can easily be remoyed to insert small experiments. One of these is Gabor holography. The chamber used in this experiment includes a specimen mount followed at 400 micron intervals by several semi-transparent resist detectors [11]. A soft $x$-ray camera will also be used in this area; the camera is designed to study large angle diffraction patterns generated by micron-sized biological specimens [12]. Both of these experiments will operate in a rough vacuum behind a silicon nitride membrane. Other experimental programs planned include Fourier Transform holography (where a zone plate creates the reference wave) and soft $\mathrm{x}-\mathrm{ray}$ contact microscopy.

\section{The Monochromator}

The output of the undulator does not have sufficient spatial or temporal coherence to be used directly for diffraction limited imaging. The function of the monochromator is to select photons which have the required temporal and spatial coherence. Our design philosophy [13] has been to achieve high spatially coherent flux with moderate temporal coherence ( 200 to 1600 waves). We use a spherical grating monochromator with fixed entrance and exit slits as shown schematically in Figure 1. Tables 1 and 2 list the important parameters of the monochromator.

Because of electron beam emittance effects, less than $1 \%$ of the radiation in the horizontal plane is spatially coherent, compared to about one-tbird of the radiation in the vertical plane. We choose wavelength dispersion in the horizontal direction because an entrance slit can be used to ensure wavelength stability at the exit slit even in the pres:nce of small electron beam movements. Upstream of the grating, the entrance slit collimates the beam. This drastically reduces the thermal load on the grating and eliminates the need for active cooling. Furthermore, the highly collimated $x$-ray beam remains in focus 
Submitted to SRI88

\begin{tabular}{|c|c|}
\hline Parameter & Value \\
\hline Grating density & 540 lines $/ \mathrm{mm}$ \\
\hline radius & $44 \mathrm{~m}$ \\
\hline size & $5 \mathrm{~cm}$ square \\
\hline land/groove ratio & 0.75 \\
\hline groove depth & 90 Angstroms \\
\hline material & nickel coated fused silica \\
\hline
\end{tabular}

Table 1. Grating Parameters

\begin{tabular}{|c|c|c|}
\hline Parameter & Short Branch & Long Branch \\
\hline Entrance arm- & $2.00 \mathrm{~m}$ & $2.00 \mathrm{~m}$ \\
\hline Exit arm & $2.36 \mathrm{~m}$ & $3.09 \mathrm{~m}$ \\
\hline Deviation angle & $5.68^{\circ}$ & $6.48^{\circ}$ \\
\hline
\end{tabular}

Table 2. Parameters for Short and Long Wavelength Branches

over a useful range of wavelengths without the need to move entrance or exit slits. Note that focusing onto the entrance slit would not provide any additional flux that could be used for diffraction limited imaging.

Our monochromator has two exit slits both placed in the inside order allowing two experiments to run simultaneously. One of the exit slits will supply radiation in the wavelength range 16 to 25 Angstroms with resolving power greater than 1000 , while the other will provide radiation in the range 20 to 50 Angstroms, also with resolving power greater than 1000. The monochromator can provide radiation down to 10 Angstroms and up to 90 Angstroms, although not at such high resolving power. In normal operation the undulator will be tuned so that the long wavelength branch will use the fundamental, while the short wavelength branch will use the second harmonic.

Radiation emerging from the two exit slits is further separated in the horizontal by reflection from a pair of sagittal focusing cylindrical mirrors at a graze angle of about $50 \mathrm{mrad}$. These mirrors provide the necessary separation for the experimental stations. Although a large fraction of the vertical beam is spatially coherent, its size 22 meters from the undulator source is much larger than that which can be used by our imaging experiments; hence the need for vertical refocusing.

\section{Beamline Components}

The Soft X-Ray Undulator [14] is a longer version of the NSLS mini-undulator whose performance was substantially in agreement with theoretical expectations [15]. Some important design parameters of the storage ring and undulator are given in Tables 3 and 4.

Following the path of the $x$ rays downstream from the undulator (see Figure 2), the beamline front end consists of the NSLS siandard isolation valve, mask, fast valve, and safety shutter. A water cooled aperture restricts the angular spread of the beam to 0.6 mrad high and 1.6 mrad wide. Just downstream of this aperture are the beam position monitors [16] which are the detectors in a closed orbit feedback system [3] which maintains 


\section{Submitted to SRI88}

\begin{tabular}{|c|c|}
\hline Parameter & Value \\
\hline Energy & $2.5 \mathrm{GeV}$ \\
\hline Maximum Current & $500 \mathrm{~mA}$ \\
\hline Horizontal size $\sigma_{x}$ & $390 \mu \mathrm{m}$ \\
\hline Horizontal divergence $\sigma_{z^{\prime}}$ & $250 \mu \mathrm{rad}$ \\
\hline Vertical size $\sigma_{y}$ & $16 \mu \mathrm{m}$ \\
\hline Vertical divergence $\sigma_{y^{\prime}}$ & $50 \mu \mathrm{rad}$ \\
\hline
\end{tabular}

Table 3. Electron Beam Parameters

\begin{tabular}{|c|c|}
\hline Parameter & Value \\
\hline Number of periods & 35 \\
\hline Period length & $8.0 \mathrm{~cm}$ \\
\hline Magnetic material & samarium cobalt \\
\hline Pole pieces & steel \\
\hline K value range & $0.1-2.5$ \\
\hline Range of fundamental & $17-70$ Angstroms \\
\hline Maximum power & 800 Watts \\
\hline Opening angle (zero emittance) & 33 microradians (at 30 Angstroms) \\
\hline Opening angle (with emittance) & 250 microradians horizontal \\
\hline & 60 microradians vertical \\
\hline
\end{tabular}

Table 4. Undulator Parameters

the direction of the $\mathrm{x}$-ray beam. The beam can be collimated by a set of four motor driven water cooled inclined jaws.

Downstream of the shield wall, a water cooled plane metal mirror [2] intercepts the beam at a graze angle of $40 \mathrm{mrad}$, deflecting it to the imaging branch, X-1A. The mirror can be retracted to provide beam to a spectroscopy branch, X-1B. The mirror is gold coated with a central beryllium stripe. The beryllium coating can be used to reject wavelengths shorter than 18 Angstroms. When shorter wavelengths are needed, the gold area can be translated into the bean. Next is a set of five interchangeable entrance slits [17]. These slits accept from 1.5 to 50 microradians and produce a beam whose divergence is approximately 60 microradians. The slits are made of molybdenum and are mounted to a heat pipe cooled copper block. Four motor driven grating masks (with heat pipe cooling) can be used for further collimation. The grating masks have been designed so they can be used as auxiliary beam position monitors in the two detector feedback configuration which constrains both the position and angle of the beam. Next in order is the spherical grating and exit slits described above.

Downstream of each of the exit slits are the refocusing mirrors which have motorized beam steering capabilities. These mirrors are about $3 \mathrm{~cm}$ square and have a cylindrical radius of about $20 \mathrm{~cm}$. The long wavelength branch refocusing mirror is coated with beryllium, while the short wavelength branch is nickel coated. The long wavelength branch has a differential pumping section downstream of the nirror which allows easy interchange 


\section{Submitted to SRI88}

of experimental components. The experimental stations are mounted on massive granite blocks which are vibration isolated from the floor with pneumatic supports.

\section{Initial results}

At the time of this Conference, the monochromator has been in use for only a week. Figure 3 shorrs the first measured output of the SXU corresponding to magnetic deflection parameters $(K)$ of 1.5 and 2.5. The output is the total yield from a gold photodiode downstream of the long wavelength branch exit slit. The gold surface of the plane mirror was in the beam. The beamline apertures accepted only 6 microradians in the horizontal and 60 microradians in the vertical, which are no larger than the rms opening angles of the radiation. The yield indicates useful intensity out past the boron $F_{1}$-edge and down to the nickel L-III edge (where the nickel coated grating becomes inefficient). The fundamental and second harmonic at a $K$ of 1.5 are both useful for imaging. Four harmonics are evident from the yield at the higher $K$ value. Note the presence of even harmonics $(\mathrm{m}=2,4, \ldots)$ on axis, an effect of the electron beam emittance [18]. Extraction of the absolute spectral intensity from these data is underway, but preliminary analysis indicates that the intensity of the harmonic peaks is within a factor of two of our expected intensity. The monochromator has already achieved a resolving power in excess of 1000 at the calcium $L$ edge near 35 Angstroms.

Closed orbit feedback was implemented in the horizontal direction only, although plans are underway to close the feedback loop in the vertical direction as well. The feedback loop seems to be working well. The loop was opened and closed without affecting other users' experiments. Further characterization of the beamline is Low underway. We anticipate that the beamline will provide high coherent power for imaging experiments by the end of 1988 .

\section{Acknowledgements}

Sam Krinksy proposed building the SXU and has been its guiding light throughout its many years of design, prototyping, construction, magnetic measurements, and conmissioning. Hank Hsieh engineered the SXU, which was designed by Dave Onsrud and John Scott. John Galayda, Lorraine Solomon, and George Stenby worked tirelessiy to make the magnetic measurements in time for installation. The beamline has had engineering help from Payman Mortazavi, Martin Woodle and Tom Oversluizen, as well as design help from Al Almasy. Ron Nawrocky designed, characterized and installed the closed orbit feedback electronics. Gerry Van Derlaske and Tony Lenhard have provided excellent technical help.

The SXU and beamline X-1A are collaborative efforts of a large fraction of the NSLS staff. We are grateful to everybody who helped.

Stony Brook is supported by the NSF under grant BBS8618065; the NSLS is supported by the DOE under contract DE-AC02-76CH000016.

\section{References}

[1] H. Rarback, C. Jacobsen, J. Kirz, and I. McNulty, in X-Ray Microscopy II, ed. D. Sayre, M. Howells, J. Kirz, H. Rarback (Springer-Verlag 1988) 10.

[2] R. DiGennaro et al. Nucl. Instr. and Meth. A266 (1988) 498. 


\section{Submitted to SRI8B}

[3] R. Nawrocky et al., ibid., 164.

[4] H. Rarback et al., Rev. Sci. Instrum. 59(1) (1988) 52.

[5] Y. Vladimirsky et al., J. Vac. Sci. Technol. B. 6(1) (1988) 311.

[6] D. Shu, D.P. Siddons, H. Rarback, and J. Kirz, Nucl. Instr. and Meth. A266 (1988) 313.

[7] S. Rothman et al., in X-Ray Microscopy II, ed. D. Sayre, M. Howells, J. Kirz, H. Rarback (Springer-Verlag 1988) 372.

[8] M. Howells et al., Science 238 (1987) 514.

(9) H. Rarback et al., in X-Ray Microscopy II, ed. D. Sayre, M. Howells, J. Kirz, H. Rarback (Springer-Verlag 1988) 194.

[10] H. Ade et al., ibid., 280.

[11] C. Jacobsen et al., ibid., 253.

[12] D. Sayre, W. Yun, and J. Kirz, ibid., 272.

[13] C. Buckley and H. Rarback, to be published.

[14] S. Krinsky, IEEE Trans. Nucl. Sci. NS-30 (1983) 3078.

[15] H. Rarback, C. Jacobsen, J. Kirz, and I. McNulty, Nucl. Instr. and Meth. A266 (1988) 96.

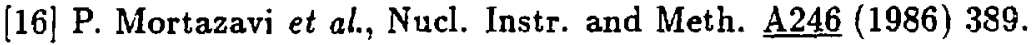

[17] D. Shu, P. Mortazavi, H. Rarback, and M. Howells, ibid., 417.

[18] C. Jacobsen and H. Rarback, in Proc. Interntl Conf on Insertion Devices for Syn. chrotron Sources, SPIE $\underline{582}$ (1985) 201.

\section{DISCLAIMER}

This report was prepared as an account of work sponsored by an agency of the United States Government. Neither the United States Government nor any agency thereof, nor any of their employees, makes any warranty, express or implied, or assumes any legal liability or responsibility for the accuracy, completeness, or usefulness of any information, apparatus, product, or process disclosed, or represents that its use would not infringe privately owned rights. Reference herein to any specific commercial product, process, or service by trade name, trademark, manufacturer, or otherwise does not necessarily constitute or imply its endorsement, recommendation, or favoring by the United States Government or any agency thereof. The views and opinions of authors expressed herein do not necessarily state or reflect those of the United States Government or any agency thereof. 


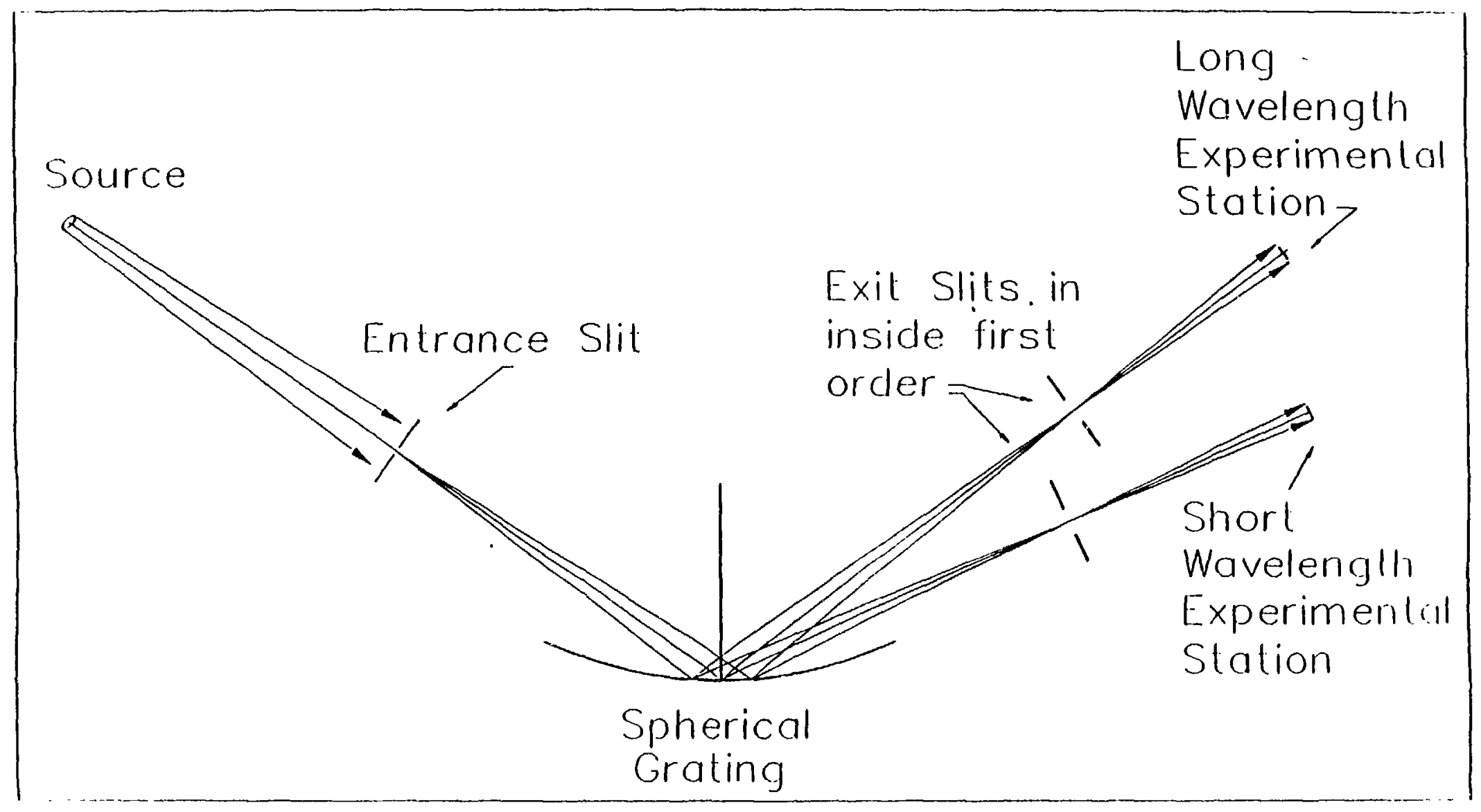

Figure 1 Schematic diagram of the beamline X-1A monochromalor. 


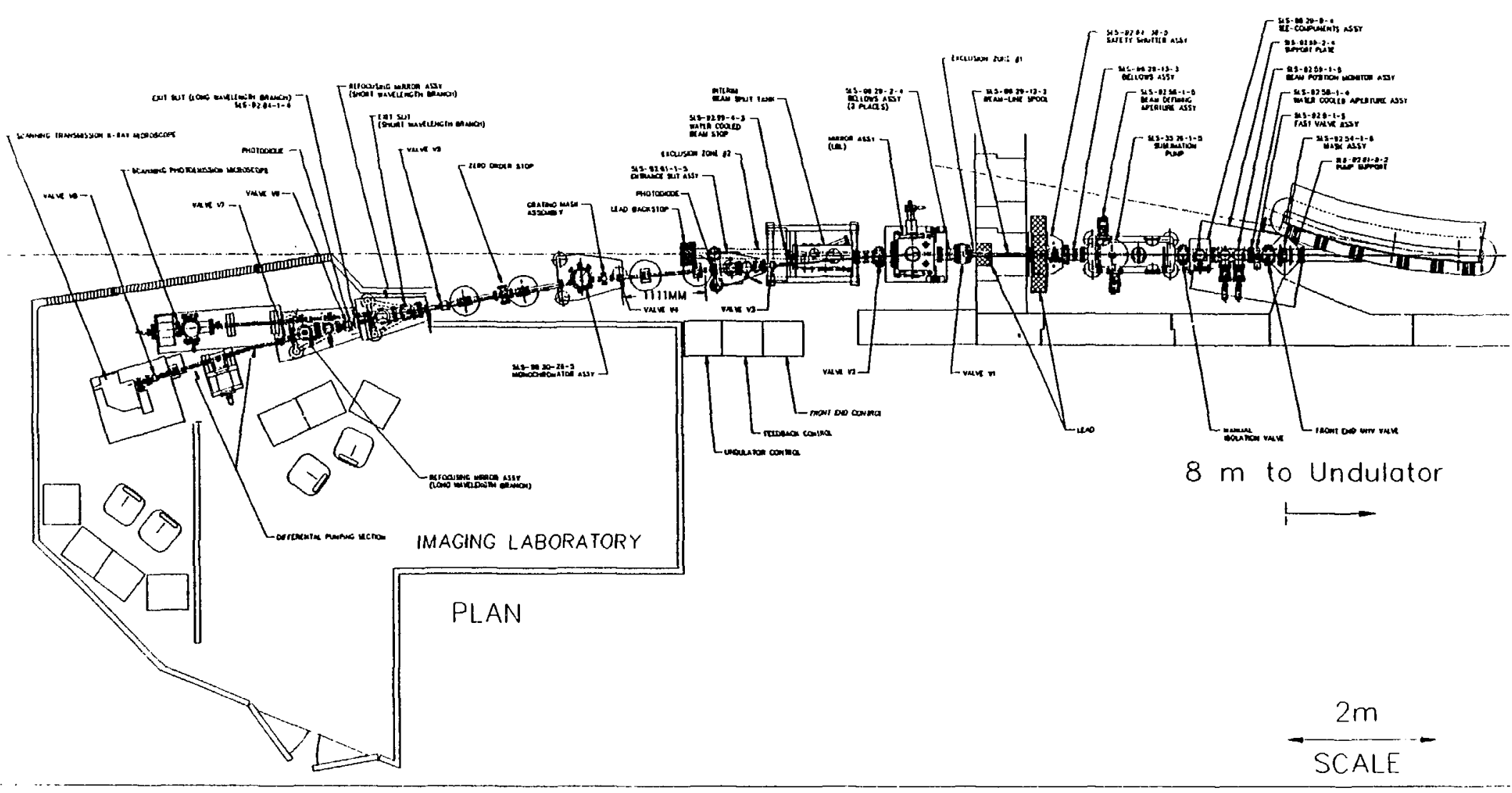

LIEVATION

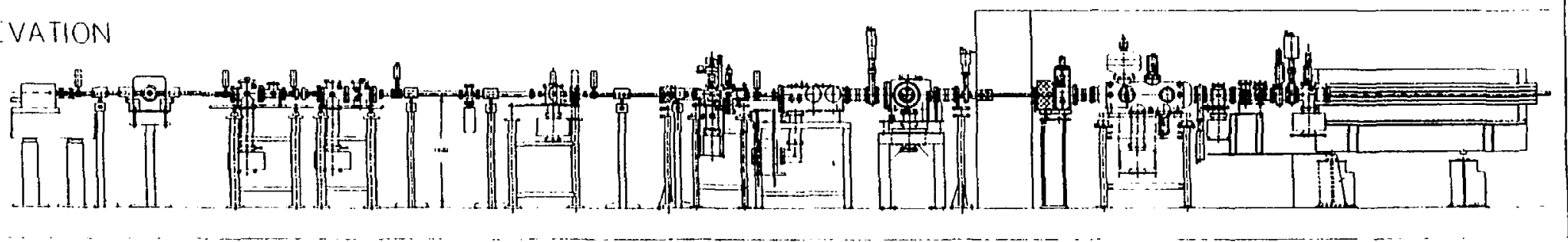

Figure 2 Drawing of beamline $\mathrm{X}-1 \mathrm{~A}$. 


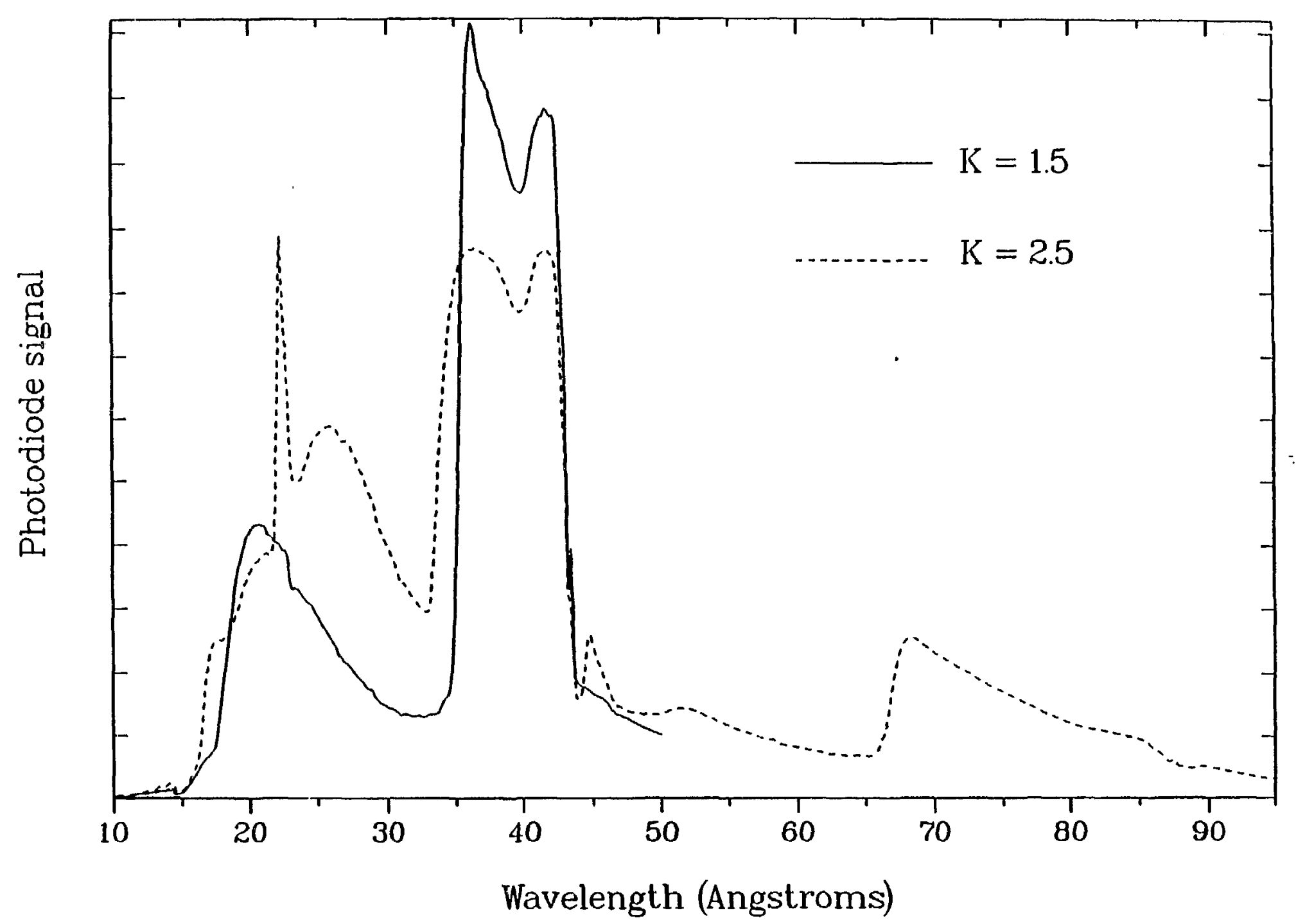

Figure 3 Output of the X-1A monochromator as measured by a gold photodiode downstream of the exit slit. Conditions for the two $K$ values were identical. The jump in the yield at 44 Angstroms is an artifact of diode contamination. See the text for further discussion. 